\title{
A Rapidly Convergent Series for Computing $\psi(z)$ and Its Derivatives
}

\author{
By Peter McCullagh
}

\begin{abstract}
We derive a series expansion for $\psi(z)$ in which the terms of the expansion are simple rational functions of $z$. From a computational viewpoint, the new series is of interest in that it converges for all $z$ not necessarily real valued, and is particularly rapid for values of $z$ near the origin. From a mathematical viewpoint the series is of interest in that, although $\psi(z)$ has poles at the negative integers and zero, the series is uniformly convergent in any finite interval $a<\operatorname{Re}(z)<b$.
\end{abstract}

A Series Expansion for $\psi(z)$. The derivative of the log gamma function, usually denoted by $\psi(z)(=d \log \Gamma(z) / d z)$, is a regular function with simple poles at the negative integers and zero. No power series can therefore have infinite radius of convergence and this constraint limits the speed at which the coefficients in such a power series decrease. In this paper we give a series expansion for $\psi(z)$ that has infinite radius of convergence despite the poles. The expansion is not a power series but converges uniformly to $\psi(z)$ in any finite interval. Convergence is particularly rapid for small $z$-say $|z|<1.0$-and, in this range, ten terms of the expansion give accuracy to at least ten decimal places. For computational purposes, the recurrence relation $\psi(z+1)=\psi(z)+1 / z$ can be used for real $z$ to obtain a value of $z$ near the origin. Extremely large values of $z$ are best dealt with by asymptotic expansions.

The Taylor series expansion for $\psi(1+z)$ about $z=0$ (Abramowitz and Stegun $[1$, Eq. 6.3.14]) is

$$
\psi(1+z)=-\gamma+\sum_{r=1}^{\infty}(-1)^{r+1} \zeta(r+1) z^{r}, \quad|z|<1,
$$

where $\gamma$ is Euler's constant, and $\zeta(r+1)=\sum_{r=1}^{\infty} k^{-r-1}$ is Riemann's zeta function. The coefficients of the power series (1) do not tend to zero, so that the speed of convergence depends entirely on the magnitude of $|z|$. To obtain a more rapidly convergent series we rewrite (1) as a double series and regroup to obtain

$$
\psi(1+z)=-\gamma+\sum_{r=1}^{\infty}(-1)^{r+1} z^{r}\left\{c_{r}+a_{r} /(z+r)\right\}, \quad|z|<\infty,
$$

where $a_{r}=r^{-r}$ and $c_{r}=\sum_{k=r+1}^{\infty} k^{-r-1}$. It is readily shown that $\lim _{r \rightarrow \infty} r^{r+1} c_{r}=$ $(e-1)^{-1} \doteq 0.582$, so that both $a_{r}$ and $c_{r}$ diminish rapidly to zero. The series (2) therefore converges pointwise for all finite $z$, and it is therefore the analytic continuation of (1). To establish uniform convergence in any finite interval $a<$ $\operatorname{Re}(z)<b$, let $g_{n}(z)$ be the sum of the first $n+1$ terms in (2) counting the constant,

Received March 3, 1980.

1980 Mathematics Subject Classification. Primary 33A15; Secondary 65D20.

(C) 1981 American Mathematical Society $0025-5718 / 81 / 0000-0020 / \$ 01.50$ 
$-\gamma$, as the first term. Then, for sufficiently large $n,(n>-a), \psi(1+z)-g_{n}(z)$ is analytic in the interval $a<\operatorname{Re}(z)<b$ and a straightforward calculation shows that

$$
\left|\psi(1+z)-g_{n}(z)\right|<2\{|z| /(n+1)\}^{n+1}
$$

for $n>\max (-a, b)$. Clearly, therefore, the series (2) is uniformly convergent in any finite interval $a<\operatorname{Re}(z)<b$. Uniformly convergent series for the derivatives of $\psi(1+z)$ follow from (2) by termwise differentiation.

For $|z| \leqslant \frac{1}{2}, g_{7}(z)$ is accurate to about 10 decimal places. For real values of $z$ outside this range, the recurrence relation can be used. If $z$ lies outside the unit circle, ten or more terms are required to give accuracy to ten decimal places. Table 1 gives values of the coefficients $\left\{c_{r}\right\}$, to twenty places of decimals.

\section{TABLE 1}

$$
\begin{aligned}
& \gamma=0.57721566490153286061 \\
& c_{1}=0.64493406684822643647 \\
& c_{2}=0.07705690315959428540 \\
& c_{3}=0.00747755469879251251 \\
& c_{4}=0.00058596630592136666 \\
& c_{5}=0.00003817924696628649 \\
& c_{6}=0.00000212260976061834 \\
& c_{7}=0.00000010277789964424 \\
& c_{8}=0.00000000440484835162 \\
& c_{9}=0.00000000016926861255 \\
& c_{10}=0.00000000000589395031 \\
& c_{11}=0.00000000000018758797 \\
& c_{12}=0.00000000000000549733 \\
& c_{13}=0.00000000000000014926 \\
& c_{14}=0.00000000000000000377 \\
& c_{15}=0.00000000000000000009
\end{aligned}
$$

Values in this table are accurate to at least 19 d.p.

A referee has pointed out that other methods are available for computing $\psi(z)$. For example, Y. L. Luke [2] gives coefficients of the Chebyshev polynomials in the expansion of $\psi(x+3)$, valid for $0 \leqslant x \leqslant 1$. For complex $z$, Luke [3] has given rational approximations for $\psi(z)+\gamma$.

The method used in this paper could in principle be applied to any function analytic except at a countable number of isolated poles. For such functions, an expansion in terms of appropriately chosen functions ought to be much more rapidly convergent than a simple power series.

\footnotetext{
Department of Mathematics

Imperial College of Science and Technology

London SW7 2BZ, England
}

1. M. Abramowitz \& I. A. Stegun, Handbook of Mathematical Functions, Dover, New York, 1970.

2. Y. L. Luke, The Special Functions and Their Approximations, Academic Press, New York, 1969.

3. Y. L. LuKE, Mathematical Functions and Their Approximations, Academic Press, New York, 1975. 